\title{
SLC3 and SLC7 families of heteromeric amino acid transporters (HATs) (version 2019.4) in the IUPHAR/BPS Guide to Pharmacology Database
}

Yoshikatsu Kanai $^{1}$

1. Osaka University, Japan

\begin{abstract}
The SLC3 and SLC7 families combine to generate functional transporters, where the subunit composition is a disulphide-linked combination of a heavy chain (SLC3 family) with a light chain (SLC7 family).
\end{abstract}

\section{Contents}

This is a citation summary for SLC3 and SLC7 families of heteromeric amino acid transporters (HATs) in the Guide to Pharmacology database (GtoPdb). It exists purely as an adjunct to the database to facilitate the recognition of citations to and from the database by citation analyzers. Readers will almost certainly want to visit the relevant sections of the database which are given here under database links.

GtoPdb is an expert-driven guide to pharmacological targets and the substances that act on them. GtoPdb is a reference work which is most usefully represented as an on-line database. As in any publication this work should be appropriately cited, and the papers it cites should also be recognized. This document provides a citation for the relevant parts of the database, and also provides a reference list for the research cited by those parts.

Please note that the database version for the citations given in GtoPdb are to the most recent preceding version in which the family or its subfamilies and targets were substantially changed. The links below are to the current version. If you need to consult the cited version, rather than the most recent version, please contact the GtoPdb curators.

\section{Database links}

SLC3 and SLC7 families of heteromeric amino acid transporters (HATs)

http://www.guidetopharmacology.org/GRAC/FamilyDisplayForward?familyld=141

\section{SLC3 family}

http://www.guidetopharmacology.org/GRAC/FamilyDisplayForward? familyld=168

Transporters

rBAT

http://www.guidetopharmacology.org/GRAC/ObjectDisplayForward?objectld=889

4F2hc

http://www.guidetopharmacology.org/GRAC/ObjectDisplayForward?objectld=890 
http://www.guidetopharmacology.org/GRAC/FamilyDisplayForward?familyld=169

Transporters

CAT1 (High affinity cationic amino acid transporter 1)

http://www.guidetopharmacology.org/GRAC/ObjectDisplayForward?objectld=891

CAT2(Low affinity cationic amino acid transporter 2)

http://www.guidetopharmacology.org/GRAC/ObjectDisplayForward?objectld=892

CAT3(Cationic amino acid transporter 3)

http://www.guidetopharmacology.org/GRAC/ObjectDisplayForward?objectld=893

CAT4(Cationic amino acid transporter 4)

http://www.guidetopharmacology.org/GRAC/ObjectDisplayForward?objectld=894

Probable cationic amino acid transporter

http://www.guidetopharmacology.org/GRAC/ObjectDisplayForward?objectld=895

LAT1(L-type amino acid transporter 1)

http://www.guidetopharmacology.org/GRAC/ObjectDisplayForward?objectld=896

LAT2(L-type amino acid transporter 2)

http://www.guidetopharmacology.org/GRAC/ObjectDisplayForward?objectld=897

$y+L A T 1(y+L$ amino acid transporter 1)

http://www.guidetopharmacology.org/GRAC/ObjectDisplayForward?objectld=898

$y+L A T 2(y+L$ amino acid transporter 2)

http://www.guidetopharmacology.org/GRAC/ObjectDisplayForward?objectld=899

$\mathrm{b}^{0,+} \mathrm{AT}\left(\mathrm{b}^{0,+}\right.$-type amino acid transporter 1$)$

http://www.guidetopharmacology.org/GRAC/ObjectDisplayForward?objectld=900

Asc-1 (Asc-type amino acid transporter 1)

http://www.guidetopharmacology.org/GRAC/ObjectDisplayForward?objectld=901

xCT(Cystine/glutamate transporter)

http://www.guidetopharmacology.org/GRAC/ObjectDisplayForward?objectld=902

AGT1

http://www.guidetopharmacology.org/GRAC/ObjectDisplayForward?objectld=903

\section{References}

1. Etoga JL, Ahmed SK, Patel S, Bridges RJ and Thompson CM. (2010) Conformationally-restricted amino acid analogues bearing a distal sulfonic acid show selective inhibition of system $\times(c)(-)$ over the vesicular glutamate transporter. Bioorg. Med. Chem. Lett. 20: 2680-3 [PMID:20303751]

2. Hayashi K and Anzai N. (2017) Novel therapeutic approaches targeting L-type amino acid transporters for cancer treatment. World J Gastrointest Oncol 9: 21-29 [PMID:28144396]

3. Hayashi K, Jutabha P, Endou H, Sagara $H$ and Anzai N. (2013) LAT1 is a critical transporter of essential amino acids for immune reactions in activated human T cells. J. Immunol. 191: 4080-5 [PMID:24038088]

4. Hayashi K, Jutabha P, Kamai T, Endou H and Anzai N. (2014) LAT1 is a central transporter of essential amino acids in human umbilical vein endothelial cells. J. Pharmacol. Sci. 124: 511-3 [PMID:24671056]

5. Hayashi K, Jutabha P, Maeda S, Supak Y, Ouchi M, Endou H, Fujita T, Chida M and Anzai N. (2016) LAT1 acts as a crucial transporter of amino acids in human thymic carcinoma cells. J. Pharmacol. Sci. 132: 201-204 [PMID:27567475]

6. Huttunen KM, Gynther M, Huttunen J, Puris E, Spicer JA and Denny WA. (2016) A Selective and Slowly Reversible Inhibitor of I-Type Amino Acid Transporter 1 (LAT1) Potentiates Antiproliferative Drug Efficacy in Cancer Cells. J. Med. Chem. 59: 5740-51 [PMID:27253989]

7. Huttunen KM, Huttunen J, Aufderhaar I, Gynther M, Denny WA and Spicer JA. (2016) L-Type amino acid transporter 1 (lat1)-mediated targeted delivery of perforin inhibitors. Int $J$ Pharm 498: 205-16 [PMID:26705152]

8. Imai H, Kaira K, Oriuchi N, Shimizu K, Tominaga H, Yanagitani N, Sunaga N, Ishizuka T, Nagamori S and Promchan $\mathrm{K}$ et al.. (2010) Inhibition of L-type amino acid transporter 1 has antitumor activity in non-small 
cell lung cancer. Anticancer Res. 30: 4819-28 [PMID:21187458]

9. Kongpracha P, Nagamori S, Wiriyasermkul P, Tanaka Y, Kaneda K, Okuda S, Ohgaki R and Kanai Y. (2017) Structure-activity relationship of a novel series of inhibitors for cancer type transporter L-type amino acid transporter 1 (LAT1). J. Pharmacol. Sci. 133: 96-102 [PMID:28242177]

10. Lippincott J, H van der Horst E, Zachwieja J and Tran H.. (2013) Anti-cd98 antibodies and methods of use thereof. Patent number: WO2013078377 A1.

11. Marshall AD, van Geldermalsen M, Otte NJ, Anderson LA, Lum T, Vellozzi MA, Zhang BK, Thoeng A, Wang $Q$ and Rasko JE et al.. (2016) LAT1 is a putative therapeutic target in endometrioid endometrial carcinoma. Int. J. Cancer 139: 2529-39 [PMID:27486861]

12. Napolitano L, Scalise M, Koyioni M, Koutentis P, Catto M, Eberini I, Parravicini C, Palazzolo L, Pisani L and Galluccio M et al.. (2017) Potent inhibitors of human LAT1 (SLC7A5) transporter based on dithiazole and dithiazine compounds for development of anticancer drugs. Biochem. Pharmacol. 143: 39-52 [PMID:28709952]

13. Nii T, Segawa H, Taketani Y, Tani Y, Ohkido M, Kishida S, Ito M, Endou H, Kanai Y and Takeda Eet al.. (2001) Molecular events involved in up-regulating human $\mathrm{Na}$-independent neutral amino acid transporter LAT1 during T-cell activation. Biochem. J. 358: 693-704 [PMID:11535130]

14. Oda K, Hosoda N, Endo H, Saito K, Tsujihara K, Yamamura M, Sakata T, Anzai N, Wempe MF and Kanai $Y$ et al.. (2010) L-type amino acid transporter 1 inhibitors inhibit tumor cell growth.Cancer Sci. 101: 173-9 [PMID:19900191]

15. Papin-Michault C, Bonnetaud C, Dufour M, Almairac F, Coutts M, Patouraux S, Virolle T, Darcourt J and Burel-Vandenbos F. (2016) Study of LAT1 Expression in Brain Metastases: Towards a Better Understanding of the Results of Positron Emission Tomography Using Amino Acid Tracers. PLOS ONE 11: e0157139 [PMID:27276226]

16. Park K. (2017) Insight into brain-targeted drug delivery via LAT1-utilizing prodrugs.J Control Release 261: 368 [PMID:28780138]

17. Puris E, Gynther M, Huttunen J, Petsalo A and Huttunen KM. (2017) L-type amino acid transporter 1 utilizing prodrugs: How to achieve effective brain delivery and low systemic exposure of drugs. J Control Release 261: 93-104 [PMID:28662899]

18. Ren W, Liu G, Yin J, Tan B, Wu G, Bazer FW, Peng Y and Yin Y. (2017) Amino-acid transporters in T-cell activation and differentiation. Cell Death Dis 8: e2655 [PMID:28252650]

19. Wolf S, Janzen A, Vékony N, Martiné U, Strand D and Closs El. (2002) Expression of solute carrier 7A4 (SLC7A4) in the plasma membrane is not sufficient to mediate amino acid transport activity. Biochem. J. 364: 767-75 [PMID:12049641]

20. Yamauchi K, Sakurai H, Kimura T, Wiriyasermkul P, Nagamori S, Kanai Y and Kohno N. (2009) System L amino acid transporter inhibitor enhances anti-tumor activity of cisplatin in a head and neck squamous cell carcinoma cell line. Cancer Lett. 276: 95-101 [PMID:19058911] 